\title{
Modelling the Transmission Dynamics of Contagious Bovine Pleuropneumonia in the Presence of Antibiotic Treatment with Limited Medical Supply
}

\author{
Achamyelesh A. Aligaz and Justin M.W. Munganga \\ Department of Mathematical Sciences, University of South Africa \\ Preller St, Muckleneuk, 0002 Pretoria, South Africa \\ E-mail(corresp.): 58548866@mylife.unisa.ac.za \\ E-mail: Mungajmw@unisa.ac.za
}

Received December 12, 2019; revised October 4, 2020; accepted October 5, 2020

\begin{abstract}
We present and analyze a mathematical model of the transmission dynamics of Contagious Bovine Pleuropneumonia (CBPP) in the presence of antibiotic treatment with limited medical supply. We use a saturated treatment function to model the effect of delayed treatment. We prove that there exist one disease free equilibrium and at most two endemic equilibrium solutions. A backward bifurcation occurs for small values of delay constant such that two endemic equilibriums exist if $\mathcal{R}_{t} \in\left(\mathcal{R}_{t}^{*}, 1\right)$; where, $\mathcal{R}_{t}$ is the treatment reproduction number and $\mathcal{R}_{t}^{*}$ is a threshold such that the disease dies out if $\mathcal{R}_{t} \leq \mathcal{R}_{t}^{*}$ and persists in the population if $\mathcal{R}_{t}>\mathcal{R}_{t}^{*}$. However, when a backward bifurcation occurs, a disease free system may easily be shifted to an epidemic. The bifurcation turns forward when the delay constant increases; thus, the disease free equilibrium becomes globally asymptotically stable if $\mathcal{R}_{t}<1$, and there exist unique and globally asymptotically stable endemic equilibrium if $\mathcal{R}_{t}>1$. However, the amount of maximal medical resource required to control the disease increases as the value of the delay constant increases. Thus, antibiotic treatment with limited medical supply setting would not successfully control CBPP unless we avoid any delayed treatment, improve the efficacy and availability of medical resources or it is given along with vaccination.
\end{abstract}

Keywords: contagious bovine pleuropneumonia, backward bifurcation, equilibria, treatment reproduction number, stability and threshold value.

AMS Subject Classification: 34D20; 34D23; 92D30.

Copyright (C) 2021 The Author(s). Published by Vilnius Gediminas Technical University

This is an Open Access article distributed under the terms of the Creative Commons Attribution License (http://creativecommons.org/licenses/by/4.0/), which permits unrestricted use, distribution, and reproduction in any medium, provided the original author and source are credited. 


\section{Introduction}

Contagious Bovine Pleuropneumonia (CBPP) is a disease of cattle caused by Mycoplasma mycoides subspecies mycoides ( $\mathrm{Mmm}$ ) small colony that infect lungs of cattle and water buffalo [24]. Transmission of the disease occurs through direct contact between an infected and a susceptible animal which becomes infected by inhaling droplets disseminated by coughing [24]. It is characterized by a relatively long incubation period, its variable course and insidious nature [19]. CBPP spread almost to all parts of the world in the nineteenth century through the cattle trade [27]. CBPP is one of the major threats to cattle health and one of the main stumbling blocks to the growth of the livestock industry on the African continent, see [8,23,31] for more details. A CBPP outbreak could cause the loss of a household's entire income from cattle keeping [13]. Different controlling mechanisms including movement control, slaughter of suspected cases [9,27] and testing strategies based on Complement Fixation Test (CFT) [4,11] has been employed to control the disease.

However, movement control, test and slaughter policies would be costly to implement and cause economic crises. Trans-human livelihoods systems are recognized as rational and environmentally friendly foundations for sustainable development of arid lands [22]. Public health situation of CBPP in Africa, most countries use vaccination, usually carried out by official veterinary services, as a form of control. The T1/44 vaccine, an attenuated live MmmSC vaccine strain, has been in use since 1956 [3]. However, vaccines were exclusively distributed by state veterinary services so that control of CBPP was very challenging in sub-Saharan Africa, as reported in [26], failure to deliver control services to farmers whose cattle are at high risk of exposure to infection was one of the policy problem for CBPP control in sub-Saharan Africa. Also, a recent study which is conducted based on the information of the 1995 outbreak of CBPP investigated that time factor it took after the previous outbreak (56 years), myths, believes, perceptions towards CBPP outbreak in the district by different ethnic groups and conflicting information disseminated by other stakeholder sources promoted lack of adoption and compliance by the community [25]. Although the use of antibiotic is against the Office International des Epizootics (OIE) Terrestrial Animal Health Code or is theoretically prohibited, antibiotic treatment is widely applied in the field for the specific treatment of CBPP, or simply to combat a range of respiratory diseases [8]. Antibiotics are already available on the market and their use has a direct impact on poverty alleviation by diminishing the mortality rate of cattle [7]. Therefore, vaccination and the controversial antibiotic treatment are assumed to be the main strategies for control of CBPP in pastoral areas [19]. However, mass vaccination programmes based on the T1/44 vaccine that are not combined with other interventions are unlikely to eradicate CBPP even when aggressively applied biannually over a five-year period [19]. Proven treatment regimes have considerable impact on the transmission of $\mathrm{CBPP}$ and reduce the effective reproductive numbers of the disease [10]. Treatment programs can reduce both the prevalence of CBPP and the morbidity, mortality and production losses associated with CBPP infection. The data on antibiotic treatment indicate that treatment may have more value 
as a CBPP control tool than current vaccines [6].

Mathematical models provide the means to generate evidence-based information on infectious disease control and play an important role in understanding the dynamics of infectious diseases [14]. Thus, the transmission dynamics of CBPP was also modeled by some researchers for different purposes. A deterministic mathematical model for the transmission of CBPP was presented to compare economic efficiency of local (i.e., at the herd level) CBPP-control strategies (vaccination and antibiotic treatments) in [15]. It revealed that antibiotics were the most economically efficient strategy than vaccination. Homogeneous and heterogeneous population models of the transmission dynamics of CBPP are also presented in [19] and [20], respectively. Both models indicated that vaccination alone with currently available vaccines was unlikely to eradicate the disease. In addition, a program involving vaccination of healthy animals with treatment of clinical cases was found to be the most promising intervention scenario [20]. In [30], modeling techniques are used to assess the potential impact of early elimination of infected cattle via accurate diagnosis on CBPP dynamics and the model predicted that regular testing and elimination of positive reactors using improved tests will play a significant role in minimizing CBPP burden especially in the situation where improved vaccines are yet to be developed. A model without any intervention is presented and elasticity analysis was done in [1] to identify the most important parameter that affect the dynamics of CBPP, it was found that effective contact rate and the rate of recovery are the top two parameters that controls the transmission dynamics of CBPP. It is known that effective contact rate and recovery rate are manageable by vaccination and antibiotic treatment, respectively. Considering results obtained in $[1,19,20,30]$, a model with vaccination and antibiotic treatment, independently and in combination was presented in [2] and it was found that combined vaccination and antibiotic treatment is a better controlling method than the independent use of vaccination and antibiotic treatment, which is in agreement with [20].

However, in the previous studies, the rate of recovery due to antibiotic treatment was taken as some constant values with simple assumptions. In this paper, to study the impact of limited medical resource supply in resources limited countries, we use a saturated treatment function $h(I)=\frac{a I}{b+I}$, where, $a \geq 0$ represents the maximal medical resources supplied per unit time and $b>0$ is half-saturation constant that measures the extent of the effect of a delay in the treatment of infectious cattle [12] or the efficiency of the medical resource supply in the sense that if $b$ is smaller, then the efficiency is higher [36].

The remaining part of this work is divided into five sections. In Section 2, we state the basic assumptions and parameter values. In Section 3, we present the mathematical model and show the well-posedness of the system. In Section 4, we find the equilibrium solutions of the system and calculate the treatment reproduction number. In Section 5, we determine the stability of disease free equilibrium (DFE) and endemic equilibrium (EE). In Section 6, we present numerical simulations and interpret the results. Lastly, in Section 7, we discuss results and conclusions. 


\section{Basic assumptions and parameters}

We assume homogeneous cattle population subdivided into susceptible, exposed, infectious, persistently infected and recovered compartments. All assumption except those related to a saturated treatment function are taken from $[19,20]$. It is assumed that all new born animals join susceptible class $(S)$ at rate $\mu$. Susceptible cattle move to the exposed compartment $(E)$ at a rate $\beta \frac{I}{N}$. Cattle in the exposed compartment move to the infectious compartment $(I)$ at a rate $\gamma$. Natural mortality is assumed to be equal to birth rate, both are denoted by $\mu$. Natural mortality results in loss from all six compartments. The infectious cattle either naturally heal at a rate $\alpha_{r}$ or they pass through a process of sequestration and enter into persistently infected $(Q)$ compartment at a rate $\alpha_{q}$ or they receive antibiotic treatment and enter directly into the recovered $(R)$ compartment at a rate $\frac{a}{b+I}$, where, $a \geq 0$ represents the maximal medical resources supplied per unit time and $b>0$ is half-saturation constant which measures the efficiency of the medical resource supply in the sense that if $b$ is smaller, then the efficiency is higher. In any intervention mechanism, efficacy and efficiency of the intervention method and proportion of the targeted population that receive the proposed treatment are basic terms that determines effectiveness of the controlling mechanism. Thus, the maximal medical resource supply per unit time, $a$, refers to the proportion of cattle that effectively receive the antibiotic treatment per unit time. Thus, the maximal medical resources supply per unit time can be defined as $a=P_{T} \epsilon_{T} ; P_{T}$ is the proportion of infectious cattle getting antibiotic treatment per unit time and $\epsilon_{T}$ is the efficacy of antibiotic that measures how effective the antibiotic is; assuming that the efficacy of the antibiotic $\left(\epsilon_{T}\right)$ is 0.75 and $40 \%$ of infectious cattle are treated $\left(P_{T}=0.4\right)$, we get $a=0.3$ meaning that the maximum proportion of infectious cattle receiving effective antibiotic treatment per a day is $30 \%$. A delay constant $b$ is assumed to be the average number of days that infectious cattle are being delayed for antibiotic treatment; that is, it refers to the average number of days that infectious cattle show clinical signs of infection before they get antibiotic treatment. It is assume that the delayed time or values of $b$ is in the range of 7 days. Cattle in persistently infected compartment are encapsulated and infected, but not infectious. As sequestra resolve and/or become non-infected, the animals in persistently infected compartment move to the recovered $(R)$ compartment at a rate $\psi$. A recovered cattle remain recovered for life. Infected sequestra can occasionally be reactive and in this instance the animal will transition from the persistently infected $(Q)$ compartment back to the infectious $(I)$ compartment at a rate $k$. The constant population size at time $t$ is given by $N=S(t)+E(t)+I(t)+Q(t)+R(t)$.

\section{A mathematical model}

We present a mathematical model of the transmission dynamics of CBPP considering antibiotic treatment within a limited medical supply setting as a controlling mechanism of CBPP. The limitation of medical supply may lead to a saturation phenomenon; that is, during severe disease outbreaks the number of 
Table 1. Description of parameters.

\begin{tabular}{ll}
\hline Parameter & Description \\
\hline$\beta$ & Effective contact rate \\
$\gamma$ & Transition rate from exposed to infectious compartment \\
$\alpha_{r}$ & Natural recovery rate of infectious cattle \\
$\alpha_{q}$ & Rate of sequestrum formation of infectious cattle \\
$k$ & Rate of sequestrum reactivation \\
$\psi$ & Rate of sequestrum resolution \\
$\mu$ & Natural mortality rate \\
$b$ & Half saturation constant \\
$\epsilon_{T}$ & Efficacy of the antibiotic \\
$P_{T}$ & Proportion of infectious cattle treated per unit time \\
$a=\epsilon_{T} P_{T}$ & Maximal medical resources supply \\
\hline
\end{tabular}

patients who need to be treated may exceed the treatment capacity so that the number of patients who receive treatment will reach a saturation level. Saturated treatment function is used and explained in details in $[12,16,21,34,35,36]$. In this model we have five compartments: susceptible $(S)$, exposed $(E)$, infectious $(I)$, persistently infected $(Q)$ and recovered $(R)$ compartments. The differential equation model for the transmission dynamics of CBPP is given by the system (3.1)-(3.6)

$$
\begin{aligned}
& \frac{d S}{d t}=\mu N-\frac{\beta S I}{N}-\mu S, \\
& \frac{d E}{d t}=\frac{\beta S I}{N}-\gamma E-\mu E, \\
& \frac{d I}{d t}=\gamma E+k Q-\alpha_{r} I-\frac{a I}{b+I}-\alpha_{q} I-\mu I, \\
& \frac{d Q}{d t}=\alpha_{q} I-k Q-\psi Q-\mu Q, \\
& \frac{d R}{d t}=\alpha_{r} I+\frac{a I}{b+I}+\psi Q-\mu R \quad \text { with } \\
& X(0)=(S(0), E(0), I(0), Q(0), R(0))=\left(S_{0}, E_{0}, I_{0}, Q_{0}, R_{0}\right) .
\end{aligned}
$$

The flow diagram of the model is shown in Figure 1.

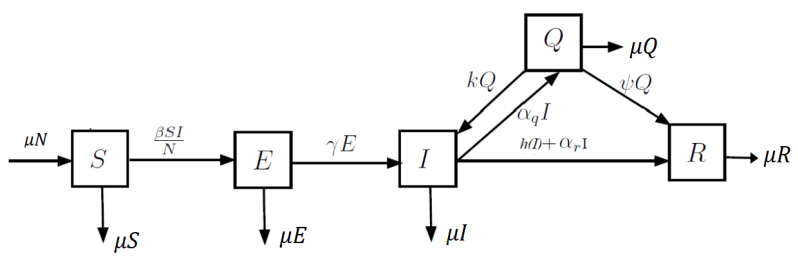

Figure 1. A compartmental model for the transmission dynamics of CBPP with antibiotic treatment, where, $h(I)=\frac{a I}{b+I}$ is a saturated treatment function. 


\subsection{Well-posedness of the system}

Theorem 1. The system (3.1)-(3.6) has a unique solution $X(t)$ which is positive and bounded provided that the initial condition, $X(0)$, is nonnegative.

Proof. The proof is classical, see [5].

\section{Equilibria and treatment reproduction number}

\subsection{Equilibrium solution of the system}

Proposition 1. The model (3.1)-(3.5) has at most three equilibrium solutions, the disease free equilibrium and at most two endemic equilibrium.

Proof. The equilibrium solution of the system is obtained by solving the equations

$$
\frac{d S}{d t}=\frac{d E}{d t}=\frac{d I}{d t}=\frac{d Q}{d t}=\frac{d R}{d t}=0
$$

We get that

$$
X^{0}=\left(S^{0}, E^{0}, I^{0}, Q^{0}, R^{0}\right)=(N, 0,0,0,0)
$$

is the disease free equilibrium. We also get equation of the form

$$
A I^{2}+B I+C=0,
$$

where $A=\beta \bar{\gamma}\left(k \alpha_{q}-\bar{\alpha} \bar{k}\right), \bar{\gamma}=\gamma+\mu, \bar{\alpha}=\alpha_{r}+\alpha_{q}+\mu, B=(b \beta \bar{\gamma}+\bar{\gamma} \mu N)\left(k \alpha_{q}-\right.$ $\bar{\alpha} \bar{k})+\bar{k} \beta(\gamma \mu N-a \bar{\gamma}), \bar{k}=k+\psi+\mu, C=b \bar{\gamma} \mu N\left(k \alpha_{q}-\bar{\alpha} \bar{k}\right)+\bar{k} \mu N(b \gamma \beta-a \bar{\gamma})$. Therefore, candidate solutions of the quadratic equation (4.2) are given by the formulas

$$
I=\frac{-B+\sqrt{B^{2}-4 A C}}{2 A}=I_{1}^{*} \quad \text { and } \quad I=\frac{-B-\sqrt{B^{2}-4 A C}}{2 A}=I_{2}^{*} .
$$

For $I_{1}^{*}>0$ and $I_{2}^{*}>0$, endemic equilibria are given by

$$
X_{1}^{*}=\left(S_{1}^{*}, E_{1}^{*}, I_{1}^{*}, Q_{1}^{*}, R_{1}^{*}\right) \quad \text { and } \quad X_{2}^{*}=\left(S_{2}^{*}, E_{2}^{*}, I_{2}^{*}, Q_{2}^{*}, R_{2}^{*}\right) .
$$

Thus, there exists a disease free equilibrium and at most two endemic equilibrium solutions.

\subsection{Treatment reproduction number, $R_{t}$}

In the presence of antibiotic treatment, we use the term treatment reproduction number, $\mathcal{R}_{t}$, instead of the commonly used basic reproduction number, $\mathcal{R}_{0}$. We used the next generation matrix, see [32], to find the treatment reproduction number. $E, I$ and $Q$ compartments are considered as disease compartments, $S$ and $R$ are non-disease compartments. Thus, the treatment reproduction number is obtained as

$$
\mathcal{R}_{t}=\frac{\gamma \beta \bar{k}}{\bar{\gamma}\left(\bar{k}(\bar{\alpha}+a / b)-k \alpha_{q}\right)}=\frac{\bar{k} \bar{\alpha}-k \alpha_{q}}{\bar{k}(\bar{\alpha}+a / b)-k \alpha_{q}} \mathcal{R}_{0},
$$


where, $\bar{k}=k+\psi+\mu, \bar{\alpha}=\alpha_{r}+\alpha_{q}+\mu, \mathcal{R}_{0}=\frac{\beta \gamma \bar{k}}{\bar{\gamma}\left(\bar{k} \bar{\alpha}-k \alpha_{q}\right)}$ is the basic reproduction number, without any intervention, as in $[1,2]$ and $\mathcal{R}_{t}$ is the treatment reproduction number which refers to the average number of secondary cases caused by an infected individual over the course of its infectious period in the presence of antibiotic treatment. Since $\frac{\bar{k} \bar{\alpha}-k \alpha_{q}}{\bar{k}(\bar{\alpha}+a / b)-k \alpha_{q}}<1, \mathcal{R}_{t}<\mathcal{R}_{0}$ and $\mathcal{R}_{t}$ reduces to $\mathcal{R}_{0}$ if $a=0$.

Proposition 2. $C=0$ if and only if $\mathcal{R}_{t}=1, C<0$ if and only if $\mathcal{R}_{t}<1$, and $C>0$ if and only if $\mathcal{R}_{t}>1$.

Proof. The proof is obvious.

Table 2 summarizes the existence of $I_{1}^{*}$ and $I_{2}^{*}$ for different values of $B$ and $C$ or $\mathcal{R}_{t}$.

Table 2. Summary of $I_{1}^{*}$ and $I_{2}^{*}$ of a CBPP model with antibiotic treatment given by (3.1)-(3.6) showing the relation between values of $\mathcal{R}_{t}$ and existence of EE.

\begin{tabular}{|c|c|c|c|c|c|c|}
\hline $\mathcal{R}_{t}$ & $C$ & B & $B^{2}-4 A C$ & $I_{1}^{*}$ & $I_{2}^{*}$ & Remark \\
\hline $\mathcal{R}_{t}=1$ & $C=0$ & $\begin{array}{l}B=0 \\
B<0 \\
B>0\end{array}$ & $\begin{array}{l}=0 \\
=B^{2} \\
=B^{2}\end{array}$ & $\begin{array}{l}0 \\
<0 \\
=0\end{array}$ & $\begin{array}{l}0 \\
0 \\
>0\end{array}$ & $\begin{array}{l}\text { No EE } \\
\text { No EE } \\
I_{2}^{*} \text { is an EE }\end{array}$ \\
\hline $\mathcal{R}_{t}<1$ & $C<0$ & $\begin{array}{l}B=0 \\
B<0 \\
B<0 \\
B>0 \\
B>0 \\
B>0\end{array}$ & $\begin{array}{l}<0 \\
\in\left[0, B^{2}\right) \\
<0 \\
=0 \\
\in\left(0, B^{2}\right) \\
<0\end{array}$ & $\begin{array}{l}\operatorname{Im} \\
<0 \\
\operatorname{Im} \\
>0 \\
>0 \\
\operatorname{Im}\end{array}$ & $\begin{array}{l}\operatorname{Im} \\
<0 \\
\operatorname{Im} \\
>0 \\
>0 \\
\operatorname{Im}\end{array}$ & $\begin{array}{l}\text { No EE } \\
\text { No EE } \\
\text { No EE } \\
\text { One EE }\left(I_{1}^{*}=I_{2}^{*}\right) \\
\text { Two EE } \\
\text { No EE }\end{array}$ \\
\hline $\mathcal{R}_{t}>1$ & $C>0$ & $\begin{array}{l}B=0 \\
B<0 \\
B>0\end{array}$ & $\begin{array}{l}>0 \\
>B^{2} \\
>B^{2}\end{array}$ & $\begin{array}{l}<0 \\
<0 \\
<0\end{array}$ & $\begin{array}{l}>0 \\
>0 \\
>0\end{array}$ & $\begin{array}{l}I_{2}^{*} \text { is an } \mathrm{EE} \\
I_{2}^{*} \text { is an EE } \\
I_{2}^{*} \text { is an } \mathrm{EE}\end{array}$ \\
\hline
\end{tabular}

Remark 1. There exist two endemic equilibria if and only if $\mathcal{R}_{t}<1, B>0$ and $B^{2}-4 A C>0$ hold simultaneously.

Theorem 2. Let $(\mu N-\beta b) /(\mu N)>1 / \mathcal{R}_{0}$ and $\mathcal{R}_{t}^{*}=\beta b /\left(\mu N\left(1+1 / \mathcal{R}_{0}-2 \sqrt{\frac{\mu N-\beta b}{\mu N \mathcal{R}_{0}}}\right)\right)$, where, $\mathcal{R}_{0}$ is the basic reproduction number without intervention. Then we have the following:

(i) Two endemic equilibrium exist if and only if $\mathcal{R}_{t} \in\left(\mathcal{R}_{t}^{*}, 1\right)$.

(ii) If $\mathcal{R}_{t}=\mathcal{R}_{t}^{*}$, then there exists a unique endemic equilibrium.

(iii) If $\mathcal{R}_{t}<\mathcal{R}_{t}^{*}$, then no endemic equilibrium exists. 
Proof.

(i) For necessity, we assume that two endemic equilibria exist. From Proposition 1 and Proposition 2, Table 2, we know that $\mathcal{R}_{t}<1, B>0$ and $B^{2}-4 A C>0$ with

$$
\begin{aligned}
B & =(b \beta \bar{\gamma}+\bar{\gamma} \mu N)\left(k \alpha_{q}-\bar{\alpha} \bar{k}\right)+\bar{k} \beta(\gamma \mu N-a \bar{\gamma}) \\
& =\beta \gamma \bar{k}\left(\mu N\left(1-1 / \mathcal{R}_{0}\right)-\beta b\left(1 / \mathcal{R}_{t}\right)\right),
\end{aligned}
$$

then $B>0$ implies $\mathcal{R}_{t}>\beta b / \mu N\left(1-1 / \mathcal{R}_{0}\right)=\mathcal{R}_{t}^{\Delta}$.

Since $\frac{\mu N-\beta b}{\mu N}>\frac{1}{\mathcal{R}_{0}}$, we have $\mathcal{R}_{t}^{\Delta}<1$ and,

$$
\begin{aligned}
B^{2}-4 A C= & \left(\bar{\gamma}\left(k \alpha_{q}-\bar{k} \bar{\alpha}\right)(\beta b-\mu N)+\beta \bar{k}(\gamma \mu N-a \bar{\gamma})\right)^{2} \\
& +4 \beta \gamma \mu N \bar{\gamma} \bar{k}\left(k \alpha_{q}-\bar{k} \bar{\alpha}\right)(\mu N-\beta b) \\
= & (\beta \gamma \bar{k})^{2}\left(\left(\mu N+\frac{\mu N}{\mathcal{R}_{0}}-\frac{\beta b}{\mathcal{R}_{t}}\right)^{2}+\frac{4 \mu N(\beta b-\mu N)}{\mathcal{R}_{0}}\right)
\end{aligned}
$$

Thus, $B^{2}-4 A C>0$ if and only if

$$
\begin{array}{r}
\left(\mu N+\frac{\mu N}{\mathcal{R}_{0}}-\frac{\beta b}{\mathcal{R}_{t}}\right)^{2}+\frac{4 \mu N(\beta b-\mu N)}{\mathcal{R}_{0}}>0 \\
\Rightarrow \mu N+\frac{\mu N}{\mathcal{R}_{0}}-\frac{\beta b}{\mathcal{R}_{t}}>\sqrt{\frac{4 \mu N(\mu N-\beta b)}{\mathcal{R}_{0}}} \text { or } \\
\mu N+\frac{\mu N}{\mathcal{R}_{0}}-\frac{\beta b}{\mathcal{R}_{t}}<-\sqrt{\frac{4 \mu N(\mu N-\beta b)}{\mathcal{R}_{0}}} .
\end{array}
$$

(4.4) and (4.5) are equivalent to

$$
\begin{aligned}
& \mathcal{R}_{t}<\frac{\beta b}{\mu N\left(1+1 / \mathcal{R}_{0}+2 \sqrt{(\mu N-\beta b) / \mu N \mathcal{R}_{0}}\right)}=\mathcal{R}_{t}^{* *} \text { and } \\
& \mathcal{R}_{t}>\frac{\beta b}{\mu N\left(1+1 / \mathcal{R}_{0}-2 \sqrt{(\mu N-\beta b) / \mu N \mathcal{R}_{0}}\right)}=\mathcal{R}_{t}^{*} .
\end{aligned}
$$

Since $\mathcal{R}_{t}^{* *}<\mathcal{R}_{t}^{\Delta}$, we use $\mathcal{R}_{t}>\mathcal{R}_{t}^{*}$ so that $B>0$ and $B^{2}-4 A C>$ hold simultaneously for some parameter values. Moreover, for $\frac{\mu N-\beta b}{\mu N}>\frac{1}{\mathcal{R}_{0}}$, we have $\mathcal{R}_{t}^{\Delta}<\mathcal{R}_{t}^{*}<1$ thus proving $\mathcal{R}_{t} \in\left(\mathcal{R}_{t}^{*}, 1\right)$.

For sufficiency, we assume that $\mathcal{R}_{t} \in\left(\mathcal{R}_{t}^{*}, 1\right)$. Thus, obviously, we have $B>0$ and $B^{2}-4 A C>0$. Hence, from Proposition 1 and Table 2, two endemic equilibrium exist. Equivalently, we say that a bifurcation occurs if and only if $\frac{\mu N-\beta b}{\mu N}>1 / \mathcal{R}_{0}$ or $b<\frac{\mu N}{\beta}\left(1-1 / \mathcal{R}_{0}\right)$.

(ii) From the proof of $(i), \mathcal{R}_{t}=\mathcal{R}_{t}^{*}$ implies $\mathcal{R}_{t}<1, B>0$ and $B^{2}-4 A C=0$. Then, from Table 2 , there exist a unique endemic equilibrium $\left(X_{1}^{*}=X_{2}^{*}\right)$. 
(iii) $\mathcal{R}_{t}<\mathcal{R}_{t}^{*}$ implies $\mathcal{R}_{t} \in\left(0, \mathcal{R}_{t}^{* *}\right] \cup\left(\mathcal{R}_{t}^{* *}, \mathcal{R}_{t}^{*}\right)$. If $\mathcal{R}_{t} \in\left(\mathcal{R}_{t}^{* *}, \mathcal{R}_{t}^{*}\right)$, it follows that $B^{2}-4 A C<0$ implying that both $I_{1}^{*}$ and $I_{2}^{*}$ does not exist. And, if $\mathcal{R}_{t} \leq \mathcal{R}_{t}^{* *}$, then $B<0$; implying that both $I_{1}^{*}$ and $I_{2}^{*}$ are negative. Therefore, there exists no endemic equilibrium if $\mathcal{R}_{t}<\mathcal{R}_{t}^{*}$.

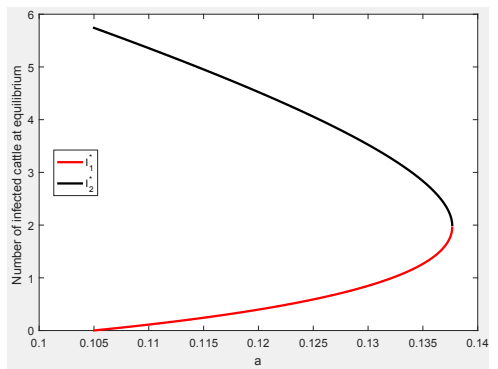

Figure 2. Plots of $I_{1}^{*}$ and $I_{2}^{*}$ for $b=1$ and $a \in(0.1049,0.1377)$ showing that $I_{1}^{*}$ and $I_{2}^{*}$ converging to 2.1210 as $a$ approaches 0.1377 . Thus, a backward bifurcation occurs if $a \in(0.1049,0.1377)$ and $b=1$.

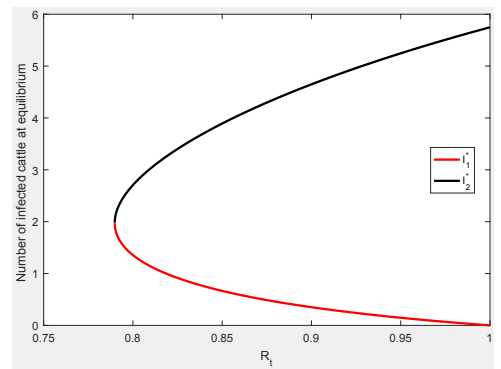

Figure 3. Plots of $I_{1}^{*}>0$ and $I_{2}^{*}>0$ for $b=1$ and $\mathcal{R}_{t} \in(0.7898,1)$ showing that $I_{1}^{*}$ and $I_{2}^{*}$ converging to 2.1210 as $\mathcal{R}_{t}$ approaches $\mathcal{R}_{t}^{*}=0.7898$. Thus, a backward bifurcation occurs if $\mathcal{R}_{t} \in(0.7898,1)$ and $b=1$.

\section{Stability analysis of equilibrium solutions}

Theorem 3. If $X^{0}$ is a DFE of the model given by equation (4.1), then $X^{0}$ is locally asymptotically stable (LAS) if $\mathcal{R}_{t} \leq 1$ and unstable if $\mathcal{R}_{t}>1$, where, $\mathcal{R}_{t}$ is the treatment reproduction number defined by equation (4.3).

Proof. The proof holds by [32, Theorem 2].

Theorem 4. If a backward bifurcation occurs and $\mathcal{R}_{t}<\mathcal{R}_{t}^{*}$, then the disease free equilibrium $X^{0}$ is globally asymptotically stable in $\Omega$. If a forward bifurcation occurs and $\mathcal{R}_{t}<1$, then the disease free equilibrium $X^{0}$ is globally asymptotically stable in $\Omega$. And, if $\mathcal{R}_{t}>1$, then the DFE is unstable, the system is uniformly persistent and there exist at least one endemic equilibrium; where, $\mathcal{R}_{t}^{*}$ is a threshold value of $\mathcal{R}_{t}$ given in Theorem $2, \Omega=\{(S, E, I, Q, R) \in$ $\left.R_{+}^{5}: S \geq 0, E \geq 0, I \geq 0, Q \geq 0, R \geq 0, S+E+I+Q+R \leq N\right\}$ is compact subset of $R^{5}$.

Proof. The system has a forward bifurcation means the DFE is the only equilibrium solution for $\mathcal{R}_{t}<1$. And, the system has a backward bifurcation means, by Theorem 2 , there does not exist any endemic equilibrium if $\mathcal{R}_{t}<\mathcal{R}_{t}^{*}<1$. Thus, it suffices to show that the DFE is globally asymptotically stable for $\mathcal{R}_{t}<1$ provided that endemic equilibrium does not exist for $\mathcal{R}_{t}<1$. For this proof we use a matrix-theoretic method explained in [29]. We assume 
$x=(E, I, Q)^{T}$ and $y=(S, R)^{T}$. Then, we set

$$
f(x, y)=(F-V) x-\mathcal{F}(x, y)+\mathcal{V}(x, y)=\left[\begin{array}{c}
\frac{\beta S^{0} I}{N}-\frac{\beta S I}{N} \\
0 \\
0
\end{array}\right]=\frac{\beta I}{N}\left[\begin{array}{c}
N-S \\
0 \\
0
\end{array}\right]
$$

where,

$$
\begin{aligned}
& \mathcal{F}=\left[\begin{array}{c}
\frac{\beta S I}{N} \\
0 \\
0
\end{array}\right], \quad \mathcal{V}=\left[\begin{array}{c}
\bar{\gamma} E \\
\left(\bar{\alpha}+\frac{a}{b+I}\right) I-\gamma E-k Q \\
k Q-\alpha_{q} I
\end{array}\right], F=\left[\begin{array}{lll}
0 & \beta & 0 \\
0 & 0 & 0 \\
0 & 0 & 0
\end{array}\right] \text { and } \\
& V=\left[\begin{array}{ccc}
\bar{\gamma} & 0 & 0 \\
-\gamma & \frac{\bar{\alpha} b+a}{b} & -k \\
0 & -\alpha_{q} & \bar{k}
\end{array}\right] \text {. } \\
& V^{-1}=\left[\begin{array}{ccc}
\frac{1}{\bar{\gamma}} & 0 & 0 \\
\frac{\gamma b \bar{k}}{\bar{\gamma}\left(\bar{k}(\bar{\alpha} b+a)-k b \alpha_{q}\right)} & \frac{b \bar{k}}{\bar{k}(\bar{\alpha} b+a)-k b \alpha_{q}} & \frac{b k}{\bar{k}(\bar{\alpha} b+a)-k b \alpha_{q}} \\
\frac{\gamma b \alpha_{q}}{\bar{\gamma}\left(\bar{k}(\bar{\alpha} b+a)-k b \alpha_{q}\right)} & \overline{\bar{k}(\bar{\alpha} b+a)-k b \alpha_{q}} & \overline{\bar{k}(\bar{\alpha} b+a)-k b \alpha_{q}}
\end{array}\right] \text { and } \\
& V^{-1} F=\beta\left[\begin{array}{ccc}
0 & \frac{1}{\bar{\gamma}} & 0 \\
0 & \frac{\gamma \bar{k}}{\bar{\gamma}\left(\bar{k}\left(\bar{\alpha}+\frac{a}{b}\right)-k \alpha_{q}\right)} & 0 \\
0 & \frac{\alpha_{q} \gamma}{\bar{\gamma}\left(\bar{k}\left(\bar{\alpha}+\frac{a}{b}\right)-k \alpha_{q}\right)} & 0
\end{array}\right]=\frac{\beta \gamma}{\bar{\gamma}}\left[\begin{array}{ccc}
0 & \frac{1}{\gamma} & 0 \\
0 & \frac{\bar{k}}{\left(\bar{k}\left(\bar{\alpha}+\frac{a}{b}\right)-k \alpha_{q}\right)} & 0 \\
0 & \frac{\alpha_{q}}{\left(\bar{k}\left(\bar{\alpha}+\frac{a}{b}\right)-k \alpha_{q}\right)} & 0
\end{array}\right] .
\end{aligned}
$$

We observe that $F \geq 0, V^{-1} \geq 0, f(x, y) \geq 0$ in $\Omega, f(x, N, 0)^{T}=0$ in $\Omega$. Since matrix $V^{-1} F$ is reducible, we use theorem 2.1 of [29] to construct a Lyapunov function. Let $\omega^{T}=\left(v_{1}, v_{2}, v_{3}\right) \geq 0$ be the left eigenvector of nonnegative matrix $V^{-1} F$ corresponding to the eigen value $\mathcal{R}_{t}$. Then

$$
\left(v_{1}, v_{2}, v_{3}\right) V^{-1} F=R_{t}\left(v_{1}, v_{2}, v_{3}\right)
$$

such that $\left(v_{1}, v_{2}, v_{3}\right) V^{-1} F=\frac{\beta \gamma}{\bar{\gamma}}(0, x, 0)$, where

$$
\begin{aligned}
& x=\frac{v_{1}}{\gamma}+\frac{\bar{k} v_{2}}{\left(\bar{k}(\bar{\alpha}+a / b)-k \alpha_{q}\right)}+\frac{\alpha_{q} v_{3}}{\left(\bar{k}(\bar{\alpha}+a / b)-k \alpha_{q}\right)}, \\
& \mathcal{R}_{t}\left(v_{1}, v_{2}, v_{3}\right)=\frac{\beta \gamma}{\bar{\gamma}}\left(\frac{\bar{k}}{\bar{k}(\bar{\alpha}+a / b)-k \alpha_{q}}\right)\left(v_{1}, v_{2}, v_{3}\right) .
\end{aligned}
$$

Thus, from equations (5.1)-(5.2), we find that $v_{1}=v_{3}=0$ and $v_{2} \in R^{+}$. 
Hence, $\omega^{T}=\left(0, v_{2}, 0\right)$. Thus, by [29, Theorem 2.1],

$$
\begin{aligned}
L & =\omega^{T} V^{-1} x \\
& =v_{2}\left(\frac{\bar{\gamma} E}{\bar{\gamma}\left(\bar{k}\left(\bar{\alpha}+\frac{a}{b}\right)-k \alpha_{q}\right)}+\frac{\bar{k} I}{\bar{k}\left(\bar{\alpha}+\frac{a}{b}\right)-k \alpha_{q}}+\frac{k Q}{\bar{k}\left(\bar{\alpha}+\frac{a}{b}\right)-k \alpha_{q}}\right) \\
& =\gamma \bar{k} E+\bar{k} \bar{\gamma} I+k \bar{\gamma} Q ; \quad \text { for } \quad v_{2}=\bar{\gamma}\left(\bar{k}\left(\bar{\alpha}+\frac{a}{b}\right)-k \alpha_{q}\right)
\end{aligned}
$$

is a Lyapunov function for the system. And,

$$
L^{\prime}=\left(\mathcal{R}_{t}-1\right) \omega^{T} x-\omega^{T} V^{-1} f(x, y)=0
$$

implies $x=0$ and $y=(N, 0)^{T}$ if $\mathcal{R}_{t}<1$ or $\mathcal{R}_{t}<\mathcal{R}_{t}^{*}<1$; therefore, $(N, 0,0,0,0)$ is the only invariant set in $\Omega$ where $L^{\prime}=0$. Thus, by LaSalle's invariance principle, the $\operatorname{DFE}(N, 0,0,0,0)$ is globally asymptotically stable in $\Omega$ provided that $\mathcal{R}_{t}<1$ and the bifurcation is forward, or if $\mathcal{R}_{t}<\mathcal{R}_{t}^{*}<1$ and the bifurcation is backward. If $\mathcal{R}_{t}>1, L^{\prime}>0$ for $x>0$ and $y=y^{0}$. By continuity, $L^{\prime}>0$ in the neighborhood of $X^{0}$; thus, solutions sufficiently close to $X^{0}$ move away from $X^{0}$, implying that $X^{0}$ is unstable. Instability of $X^{0}$ implies uniform persistence of the system. Uniform persistence and the positive invariance of the compact set $\Omega$ imply the existence of at least one EE of the system.

Theorem 5. If $\mathcal{R}_{t}>1$, then there exist unique and globally asymptotically stable endemic equilibrium in the interior of $\Omega$, where, $\Omega=\{(S, E, I, Q, R) \in$ $\left.R_{+}^{5}: S \geq 0, E \geq 0, I \geq 0, Q \geq 0, R \geq 0, S+E+I+Q+R \leq N\right\}$ is compact subset of $R^{5}$.

\section{Proof. See Appendix A.}

\section{Numerical simulations}

All parameter values except those in saturated treatment functions are taken from [19, Table 1 and Sections 2.2 and 2.3]. Assuming that natural death rate and birth rate are equal and the life expectancy of cattle is in average 5 years, natural death rate and birth rate are given by $\mu=\frac{1}{5 \times 365}$. The mode value effective contact rate is taken to be 0.126 with maximum and minimum values 0.13 and 0.07 , respectively. The incubation period is between 4 and 8 weeks with mean value of 6 weeks yields $\gamma=\frac{1}{6 \times 7}$. Without applying antibiotic treatment, the infection period is between 6 and 10 weeks with mean value of 8 weeks and $\alpha_{q}=3 \alpha_{r}$ gives $\alpha_{r}=\frac{1}{4 \times 56}$. The persistently infected period is given in a range of $18-21$ weeks with an average period of 19 weeks with 4 months $\times$ 2 reactivations per month for 582 cases gives $k=0.00009$ and $\psi=0.0075$. We assume that the efficacy of antibiotic treatment, $\epsilon_{T}$, is 0.75 . For a saturation function, $h(I)=\frac{a I}{b+I}$, we define the maximal medical resources supplied per unit time as: $a=P_{T} \epsilon_{T}$, where $P_{T}$ is the proportion of infectious cattle to be treated per unit time and $\epsilon_{T}$ is the efficacy of antibiotic that measure how 
effective the antibiotic is. Values of delay constant $b$ are assumed to be in the range of 7 days. Global sensitivity analysis of $\mathcal{R}_{t}$ to the parameters it depends on is performed using Latin hypercube sampling-partial rank correlation coefficient (LHS-PRCC) and scatter plotting methods which are explained and used in [33], these methods are carried out using MATLAB. Parameters with PRCC values are summarized in Table 3.

Table 3. Description of model parameters and their values with sensitivity index; indicating baselines, ranges and references. Units are days ${ }^{-1}$ unless otherwise defined. ${ }^{*}$ constants. P: Parameters.

\begin{tabular}{|c|c|c|c|c|}
\hline $\mathrm{P}$ & Description & $\begin{array}{l}\text { Sensitivity } \\
\text { Index }\end{array}$ & $\begin{array}{l}\text { Baseline } \\
\text { value }\end{array}$ & $\begin{array}{l}\text { Value range and } \\
\text { references }\end{array}$ \\
\hline$b$ & $\begin{array}{l}\text { Half saturation } \\
\text { constant } *\end{array}$ & 0.6463 & 2 & 0 to 7 [assumed] \\
\hline$a$ & $\begin{array}{l}\text { Maximal medical } \\
\text { resources supply } \\
\text { per unit time }\end{array}$ & -0.6420 & 0.375 & 0 to 0.75 [assumed] \\
\hline$\beta$ & Effective contact rate & 0.1429 & 0.126 & 0.07 to $0.13[19]$ \\
\hline$\alpha_{r}$ & $\begin{array}{l}\text { Natural recovery rate } \\
\text { of infectious cattle }\end{array}$ & -0.0308 & 0.0045 & 0.0036 to $0.0059[19]$ \\
\hline$\psi$ & $\begin{array}{l}\text { Rate of sequestrum } \\
\text { resolution }\end{array}$ & 0.0274 & 0.0075 & 0.0068 to $0.0079[19]$ \\
\hline$\gamma$ & $\begin{array}{l}\text { Transition rate from } \\
\text { exposed to infectious } \\
\text { compartment }\end{array}$ & -0.0235 & 0.024 & 0.018 to $0.036[19]$ \\
\hline$\mu$ & Mortality rate & 0.0141 & 0.00055 & 0.00050 to $0.00062[19]$ \\
\hline$k$ & $\begin{array}{l}\text { Rate of sequestrum } \\
\text { reactivation }\end{array}$ & 0.0109 & 0.00009 & 0.00007 to 0.00011 [19] \\
\hline$\alpha_{q}$ & $\begin{array}{l}\text { Rate of sequestrum } \\
\text { formation of } \\
\text { infectious cattle }\end{array}$ & 0.0001 & 0.013 & 0.011 to $0.018[19]$ \\
\hline$\epsilon_{T}$ & Efficacy of antibiotic $*$ & - & 0.75 & [assumed] \\
\hline$P_{T}$ & Proportion treated & - & - & $>0$ \\
\hline
\end{tabular}

It can be seen from Figure 4 that the supply of medical resources, $a$, and the half saturation constant, $b$, have the most global influence on $\mathcal{R}_{t}$ followed by the effective contact rate, $\beta$. Sensitivity index or PRCC values indicate that the value of treatment reproduction number can be decreased mainly by increasing the amount of medical resources supply and by decreasing half saturation constant or delayed treatment. Minimizing effective contact, $\beta$, also decreases the value of $\mathcal{R}_{t}$ to some extent.

Figures 5-8 are scatter plots indicating the correlation between treatment reproduction number and the parameters $a, b, \beta$ and $\alpha_{r}$, respectively.

It can be seen from Figures 5-8 that the scatter plots for $a$ and $b$ have the most visible patterns implies that the correlations between $\mathcal{R}_{t}$ and parameters 


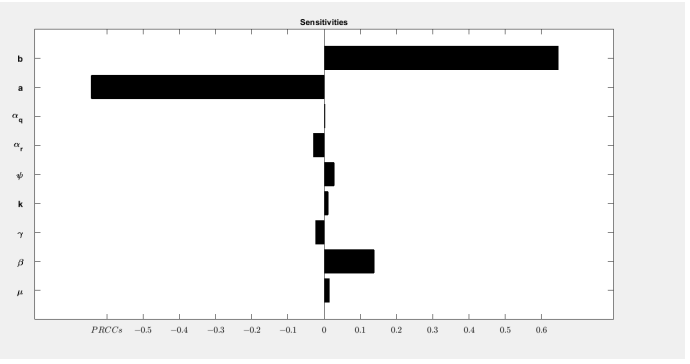

Figure 4. Sensitivity of treatment reproduction number to parameters it depends on.

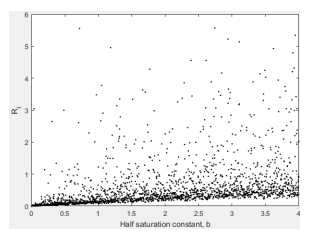

Figure 5. The scatter plot is having a pattern indicating that the correlation between $\mathcal{R}_{t}$ and $b$ is strong implying that $\mathcal{R}_{t}$ is most sensitive to $b$.

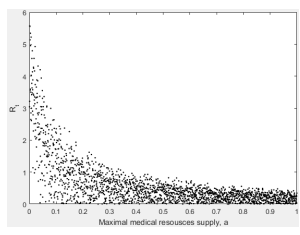

Figure 6. The scatter plot is having a visible pattern indicating that the correlation between $\mathcal{R}_{t}$ and $a$ is strong implying that $\mathcal{R}_{t}$ is also very sensitive to $a$.

$b$ and $a$ are very strong. However, the scatter plots for $\beta$ and $\alpha_{r}$ have poor patterns implies that correlations between $\mathcal{R}_{t}$ and the two parameters $\beta$ and $\alpha_{r}$ are week. Thus, parameter $b$ has the most significant influence on the value of $\mathcal{R}_{t}$, followed by $a$, but, $\beta$ and $\alpha_{r}$ have poor influence on the value of $\mathcal{R}_{t}$.

Figures 9 and 10 show the dynamics of the system when a forward bifurcation and a backward bifurcation occurs, respectively.

Figure 9 shows that the system converges to its DFE for $\mathcal{R}_{t}=0.9243<1$. However, Figure 10 shows that the system converges to it endemic equilibrium with $\mathcal{R}_{t}=0.6542<1$. In this case, a backward bifurcation occurs with a threshold $\mathcal{R}_{t}^{*}=0.1161$.

For $b=1$, Figures 2-3 show that two endemic equilibriums exist for $a \in$ $(0.1049,0.1377)$ or $\mathcal{R}_{t} \in(0.7898,1)$ implying that the disease persists in the population if the maximal medical resource supply is below 0.1377 . Thus, it is necessary to keep $a \geq 0.1377$ to control the disease. $a=P_{T} \epsilon_{T}=0.1377$ gives $P_{T}=0.1836$ implying that at least $18.36 \%$ of infectious cattle should get antibiotic treatment per a day, to control the disease. And, a forward bifurcation occurs if $b=2$, thus, $\mathcal{R}_{t}=1$ or $a=0.2098$ acts as a sharp threshold implying that at least $27.97 \%$ of infectious cattle should be treated per unit time to control the disease. Similarly, if $b=7, \mathcal{R}_{t}=1$ or $a=0.7344$ acts as a sharp threshold implying that at least $73.44 \%$ of infectious cattle should be treated per unit time to control the disease. Here, we notice that the maximal medical resource supply $(a)$ required to control the diseases increases as the value of $b$ increases. 


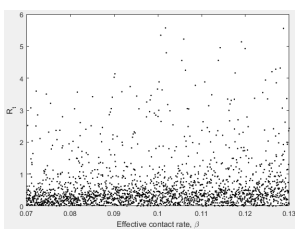

Figure 7. The scatter plot is having relatively poor pattern than Figures $5-6$ indicating that the correlation between $\mathcal{R}_{t}$ and $\beta$ is week implying that $\mathcal{R}_{t}$ is poorly sensitive to $\beta$.

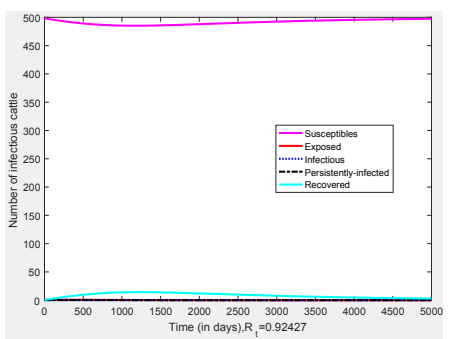

Figure 9. Number of cattle in each compartment with baseline parameter values in Table 3 except $a=0.23$ and with initial values $I_{0}=1, S_{0}=499$ and $V_{0}=E_{0}=Q_{0}=R_{0}=0$ converging to its $\operatorname{DFE}\left(S^{0}=500\right.$, $\left.E^{0}=I^{0}=Q^{0}=R^{0}=0\right)$. The system has a forward bifurcation for these parameter values with $\mathcal{R}_{t}=0.9243$.

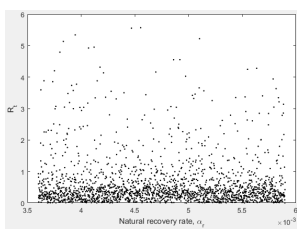

Figure 8. The scatter plot is having poor pattern indicating that the correlation between $\mathcal{R}_{t}$ and $\alpha_{r}$ is week implying that $\mathcal{R}_{t}$ is poorly sensitive to $\alpha_{r}$.

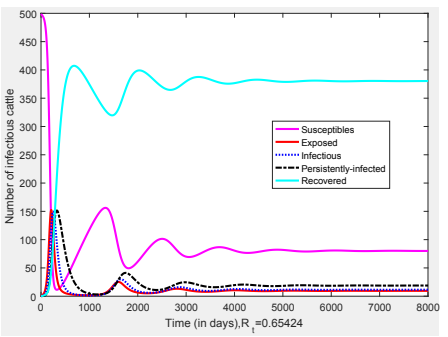

Figure 10. Number of infectious cattle with initial values $I_{0}=1$, $S_{0}=499$ and $V_{0}=E_{0}=Q_{0}=$ $R_{0}=0$ and with baseline parameter values in Table 3 except $b=0.1$ and $a=0.017$. A backward bifurcation occurs for these parameter values with $\mathcal{R}_{t}^{*}=0.1161<\mathcal{R}_{t}=0.6542<1$; the system converges to its $\operatorname{EE}\left(S^{\star}=80\right.$, $\left.E^{\star}=9, I^{\star}=11, Q^{\star}=19, R^{\star}=380\right)$.

\section{$7 \quad$ Discussion and conclusions}

In our model, we used a saturation treatment function, $h(t)=\frac{a I}{b+I}$, where, $a$ is the maximum medical resource supply and $b>0$ is a saturation constant, to model the effect of delayed treatment in controlling CBPP such that infectious cattle get antibiotic treatment and move to recovered compartment at a rate $\frac{a}{b+I}$. The system has a disease free equilibrium and at most two endemic equilibrium solutions. A backward bifurcation occurs for small delayed constant, $b<\frac{\mu N}{\beta}\left(1-\frac{1}{\mathcal{R}_{0}}\right)=1.8521=b^{*}$ such that two endemic equilibria exist if $\mathcal{R}_{t} \in\left(\mathcal{R}_{t}^{*}, 1\right)$, where, $\mathcal{R}_{t}$ is the treatment reproduction number and $\mathcal{R}_{t}^{*}$ is the threshold value of $\mathcal{R}_{t}$ given by (4.3). In this case, the disease goes extinct if $\mathcal{R}_{t}<\mathcal{R}_{t}^{*}<1$ and invades the population if $\mathcal{R}_{t}>\mathcal{R}_{t}^{*}$. However, when a backward bifurcation occurs, a stable endemic equilibrium co-exsit with a stable disease free equilibrium for $\mathcal{R}_{t}^{*}<\mathcal{R}_{t}<1$ so that disease free state may shift to the epidemic state for small perturbation of the system; that means, missing to treat few infected cattle or any delayed treatment may cause epidemic in the population. And, if $b>b^{*}$, the bifurcation turns out forward and hence 
the disease free equilibrium remain globally asymptotically stable if $\mathcal{R}_{t}<1$, and there exist unique and globally asymptotically stable endemic equilibrium if $\mathcal{R}_{t}>1$. However, the maximal medical resource supply required to control the diseases increases as the delay constant $b$ increases.

A backward bifurcation occurs for $b=1$ with threshold value $\mathcal{R}_{t}^{*}=0.7898$ or $a=0.1377$ such that two endemic equilibrium exist if $a \in(0.1049,0.1377)$ or $\mathcal{R}_{t} \in(0.7898,1)$, as shown in Figures $2-3 . \quad a=P_{T} \epsilon_{T} \geq 0.1377$ gives $P_{T} \geq$ 0.1836 meaning that at least $18.36 \%$ of infectious cattle should be treated per day to control the disease. And, for $b=2$, a forward bifurcation occurs so that $a=0.2098$ or $\mathcal{R}_{t}=1$ is a sharp threshold between the disease dying out and causing an epidemic. Where, $a=0.2098$ gives $P_{T}=0.2797$ meaning that at least $27.97 \%$ of infectious cattle should get antibiotic treatment per day to control the disease. Similarly, for $b=7$, a forward bifurcation occurs so that $a=0.7344$ or $\mathcal{R}_{t}=1$ is a sharp threshold between the disease dying out and causing an epidemic. Where, $a=0.7344$ gives $P_{T}=0.9792$ implying that at least $97.92 \%$ of infectious cattle should get antibiotic treatment per day to control the disease.

Global sensitivity analysis also show that decreasing $b$ and increasing $a$ are most important measures to decrease the treatment reproduction number and hence to control the disease. However, because of lack of awareness and poverty, decreasing delay constant, $b$, or taking cattle to treatment as soon as they are infected is probably not possible in developing countries. And, for very small values of $b$, the system will experiences a backward bifurcation and hence a stable disease free equilibrium coexist with a stable endemic equilibrium when $\mathcal{R}_{t} \in\left(\mathcal{R}_{t}^{*}, 1\right)$, thus, a disease free state may shift to epidemic for small perturbation. Meaning, the disease may persist in the population if there is any delayed treatment. And, when a forward bifurcation occurs, the amount of medical resources required to control the disease is large, but, the availability of medical resource is limited in developing countries and most cattle owners are economically poor to buy antibiotic for their cattle. Thus, it is very challenging to control CBPP using antibiotic treatment unless we avoid any delayed treatment and improve the supply and efficacy of the antibiotic. And, sensitivity analysis indicate that decreasing effective contact rate, $\beta$, is one way of decreasing the value of treatment reproduction number, next to half saturation constant and maximal medical resources, thus, we should also use vaccination along with antibiotic treatment so as to effectively control the disease as suggested in $[2,20]$.

\section{References}

[1] A.A. Aligaz and J.M.W. Munganga. Analysis of a mathematical model of the dynamics of contagious bovine pleuropneumonia. In Mathematical Methods and Models in Biosciences, Biomath Forum, Sofia, pp. 64-80, Sofia, 2018. https://doi.org/10.11145/texts.2017.12.253.

[2] A.A. Aligaz and J.M.W. Munganga. Mathematical modelling of the transmission dynamics of contagious bovine pleuropneumonia with vaccination and 
antibiotic treatment. Journal of Applied Mathematics, 2019(4):10, 2019. https://doi.org/10.1155/2019/2490313.

[3] W. Amanfu. Contagious bovine pleuropneumonia (lung sickness) in Africa. Onderstepoort Journal of veterinary Research, 76(1):13-17, 2009. https://doi.org/10.4102/ojvr.v76i1.55.

[4] A.D. Campbell and A.W. Turner. Studies of contagious bovine pluerophnuemonia of cattle. an improved complementfixation test. Australian Veterinary Journal, 4(29), 1953. https://doi.org/10.1111/j.1751-0813.1953.tb05259.x.

[5] T. Caraballo and X. Han. Applied Nonautonomous and Random Dynamical Systems: Applied Dynamical Systems. Springer, 2016. https://doi.org/10.1007/9783-319-49247-6.

[6] FAO. Towards sustainable CBPP control programmes for Africa. FAO-OIEAU/IBAR-IAEA Consultative Group on Contagious Bovine Pleuropneumonia third meeting, Rome, 2003.

[7] FAO. CBPP control: Antibiotics to the rescue? FAO-OIE-AU/IBAR-IAEA Consultative Group Meeting on CBPP in Africa, Rome, 2006.

[8] FAO. Can contagious bovine pleuropneumonia (CBPP) be eradicated? FAOOIE-AU/IBAR-IAEA Consultative group on CBPP fifth meeting, Rome, 2015.

[9] J.A. Hammond and D. Branagan. Contagious bovine pleuropneumonia in Tanganyika. Bulletin of Epizootic Disease in Africa, 13:121-147, 1965.

[10] O.J. Hubschle, R.D. Ayling, K. Godinho, O.T. Lukhele, G. Ipura-Zaire, T.G. Rowan and R.A.J. Nicholas. Danifloxacine(advocin) reduces the spread of contagious bovine pleuropneumonia to healthy in-contact cattle. Res. Vet.Sc., 81:304309, 2009. https://doi.org/10.1016/j.rvsc.2006.02.005.

[11] J.E. Huddart. Bovine contagious pleuropneumonia - a new approach to field control in Kenya. Veterinary Record, 72:1253-1254, 1960.

[12] W. Jinliang, L. Shengqiang, Z. Baowen and T. Yasuhiro. Qualitative and bifurcation analysis using an SIR model with a saturated treatment function. Mathematical and Computer Modelling, 55(3):710-722, 2012. https://doi.org/10.1016/j.mcm.2011.08.045.

[13] S.W. Kairu-Wanyoike, N.M. Taylor, C. Heffernan and H. Kiara. Micro-economic analysis of the potential impact of contagious bovine pleuropneumonia and its control by vaccination in Narok district of Kenya. Livestock Science, 197:61-72, 2017. https://doi.org/10.1016/j.livsci.2017.01.002.

[14] M.J. Keeling and P. Rohani. Modeling infectious diseases in humans and animals. Princeton University Press, 2008. https://doi.org/10.1515/9781400841035.

[15] M. Lesnoff, G. Laval, P. Bonnet and A. Workalemahu. A mathematical model of contagious bovine pleuropneumonia (CBPP) within-herd outbreaks for economic evaluation of local control strategies : an illustration from a mixed crop-livestock system in Ethiopian highlands. Animall Research, EDP Sciences, 53:429-438, 2004. https://doi.org/10.1051/animres:2004026.

[16] C.H. Li and A.M. Yousef. Bifurcation analysis of a network-based SIR epidemic model with saturated treatment function. Chaos: An Interdisciplinary Journal of Nonlinear Science, 29(3):033129, 2019. https://doi.org/10.1063/1.5079631.

[17] M.Y. Li and J.S. Muldowney. On R.A. Smith's autonomous convergence theorem. Rocky Mountain Journal of Mathematics, 25(1):365-378, 031995. https://doi.org/10.1216/rmjm/1181072289. 
[18] M.Y. Li and J.S. Muldowney. A geometric approach to global-stability problems. SIAM Journal on Mathematical Analysis, 27(4):1070-1083, 1996. https://doi.org/10.1137/S0036141094266449.

[19] J.C. Mariner, J. McDermott, J.A.P. Heesterbeek, G. Thomson and S.W. Martin. A model of contagious bovine pleuropneumonia transmission dynamics in East Africa. Preventive veterinary medicine, 73(1):55-74, 2006. https://doi.org/10.1016/j.prevetmed.2005.09.001.

[20] J. McDermott, J.C. Mariner, J.A.P. Heesterbeek, G. Thomson, P.L. Roeder and S.W. Martin. A heterogeneous population model for contagious bovine pleuropneumonia transmission and control in pastoral communities of East Africa. Preventive Veterinary Medicine, 73(1):75-91, 2006. https://doi.org/10.1016/j.prevetmed.2005.09.002.

[21] R. Naji and B. Abdulateef. The dynamics of model with nonlinear incidence rate and saturated treatment function. Science International, 29:1223-1236, 11 2017.

[22] M. Niamir-Fuller. A review of recent literature on pastoralism and transhumance in Africa. Intermediate Technology publications, London, pp. 18-46, 1999. https://doi.org/10.3362/9781780442761.002.

[23] R. Nicholas, R. Ayling and L. McAuliffe. Mycoplasma Diseases of Ruminants. CAB books. CABI, 2008. https://doi.org/10.1079/9780851990125.0000.

[24] OIE. Contagious bovine pleuropneumonia. General disease information sheet.

[25] T.T. Olekae and P.R. Naledi. Communicating livestock disease risks in Ngamiland: the case of contagious bovine pleuropneumonia. South African Geographical Journal, 101(2):192-209, 2019. https://doi.org/10.1080/03736245.2019.1581080.

[26] J.O. Onono, B. Wieland, A. Suleiman and J. Rushton. Policy analysis for delivery of contagious bovine pleuropneumonia control strategies in subSaharan Africa. OIE Revue Scientifique et Technique, 36:195-205, 042017. https://doi.org/10.20506/rst.36.1.2621.

[27] A. Provost, P. Perreau, A. Breard, C. Le Goff, J.L. Martel, G.S. Cottew and C. Le Goff. Contagious bovine pluerophnuemonia. Revue Scientifique at Technique, Office International des Epizooties, 6:625-679, 1987. https://doi.org/10.20506/rst.6.3.306.

[28] H.M. Robert. Logarithmic norms and projections applied to linear differential systems. Journal of Mathematical Analysis and Applications, 45(2):432-454, 1974. https://doi.org/10.1016/0022-247X(74)90084-5.

[29] Z. Shuai and P. van den Driessche. Global stability of infectious disease models using Lyapunov functions. SIAM Journal on Applied Mathematics, 73(4):15131532, 2013. https://doi.org/10.1137/120876642.

[30] A. Ssematimba, J. Jores and J.C. Mariner. Mathematical modelling of the transmission dynamics of contagious bovine pleuropneumonia reveals minimal target profiles for improved vaccines and diagnostic assays. PLOS ONE, 10(2):e0116730, 2015. https://doi.org/10.1371/journal.pone.0116730.

[31] N.E. Tambi, W.O. Maina and C. Ndi. An estimation of the economic impact of contagious bovine pleuropneumonia in Africa. Revue scientifique et technique (International Office of Epizootics), 25(3):999-1011, 2006. https://doi.org/10.20506/rst.25.3.1710. 
[32] P. van den Driessche and J. Watmough. Reproduction numbers and subthreshold endemic equilibria for compartmental models of disease transmission. Mathematical Biosciences, 180:29-48, 2002. https://doi.org/10.1016/S00255564(02)00108-6.

[33] J. Wu, R. Dhingra, M. Gambhir and J.V. Remais. Sensitivity analysis of infectious disease models: methods, advances and their application. Journal of The Royal Society Interface, 10(86), 2013. https://doi.org/10.1098/rsif.2012.1018.

[34] J. Zhang, J. Jia and X. Song. Analysis of an SEIR epidemic model with saturated incidence and saturated treatment function. In The Scientific World Journal, 2014. https://doi.org/10.1155/2014/910421.

[35] X. Zhang and X. Liu. Backward bifurcation of an epidemic model with saturated treatment function. Journal of Mathematical Analysis and Applications, 348(1):433-443, 2008. https://doi.org/10.1016/j.jmaa.2008.07.042.

[36] L. Zhou and M. Fan. Dynamics of an SIR epidemic model with limited medical resources revisited. Nonlinear Analysis: Real World Applications, 13(1):312-324, 2012. https://doi.org/10.1016/j.nonrwa.2011.07.036.

\section{Appendix A: Proof of Theorem 5}

Proof. Since the recovered population, $R$, can be determined from $S, E, I, Q$ and $N$, it suffices to consider the system (3.1)-(3.4) only. In this case, $\Omega=$ $\left\{(S, E, I, Q) \in R_{+}^{4}: S \geq 0, E \geq 0, I \geq 0, Q \geq 0, S+E+I+Q \leq N\right\}$ is compact subset of $R^{4}$. Now, we proof using a geometric approach introduced in [18] by showing that the following conditions of [18, Theorem 3.5] are satisfied:

(i) $\Omega$ is simply connected,

(ii) There is a compact absorbing set $K \subset \Omega$,

(iii) $X_{2}^{*}$ is the only equilibrium of the system (3.1)-(3.4) in $\Omega$ and

(iv) $\bar{q}_{2}=\lim \sup _{t \rightarrow \infty} \sup _{x_{0} \in K} \frac{1}{t} \int_{0}^{t} \mu\left(B\left(x\left(t, x_{0}\right)\right)\right) d t<0$, where, $B=P_{f} P^{-1}+$ $P J^{[2]} P^{-1}, P$ is a nonsingular $6 \times 6$ matrix valued function $x \rightarrow P(x)$ which is $C^{1}$ in $\Omega,|\cdot|_{\infty}$ defined by $|(u, v, w, x, y, z)|_{\infty}=\max \{|u|,|v|,|w|,|x|,|y|,|z|\}$ is a vector norm on $R^{6}, \mu$ is the Lozinskii measure with respect to $|\cdot|, P_{f}=(D P)(f)$ or, equivalently, $P_{f}$ is the matrix obtained by replacing each entry $p_{i j}$ in $P$ by its directional derivative in the direction of $f$ and $J^{[2]}$ is the second additive compound matrix of $J$ as defined in [17, Table 1].

For (i), obviously, $\Omega$ is simply connected in $R^{4}$. For (ii), the instability of the disease-free equilibrium implies the uniform persistence of system (3.1)-(3.4), and uniform persistence is equivalent to the existence of a compact absorbing set $K$ in the interior of $\Omega$ as $\liminf _{t \rightarrow \infty}|S(t), E(t), I(t), Q(t)|>c$ for some $c>0$. For (iii), from Table 2 , the system has a unique endemic equilibrium in the interior of $\Omega$ when $\mathcal{R}_{t}>1$. For (iv), the Jacobian matrix $\mathrm{J}$ of the system 
is given by

$$
\begin{aligned}
& J=\left[\begin{array}{ccccc}
-\beta I / N-\mu & 0 & & -\beta S / N & 0 \\
\beta I / N & -(\gamma+\mu) & \beta S / N & 0 \\
0 & \gamma & -\left(\bar{\alpha}+a b /(b+I)^{2}\right) & k \\
0 & 0 & \alpha_{q} & -\bar{k}
\end{array}\right], \\
& J^{[2]}=\left[\begin{array}{cccccc}
J_{11}^{[2]} & \beta S / N & 0 & \beta S / N & 0 & 0 \\
\gamma & J_{22}^{[2]} & k & 0 & 0 & 0 \\
0 & \alpha_{q} & J_{33}^{[2]} & 0 & 0 & -\beta S / N \\
0 & \beta I / N & 0 & J_{44}^{[2]} & k & 0 \\
0 & 0 & \beta I / N & \alpha_{q} & J_{55}^{[2]} & \beta S / N \\
0 & 0 & 0 & 0 & \gamma & J_{66}^{[2]}
\end{array}\right],
\end{aligned}
$$

where, $J_{11}^{[2]}=-\left(\frac{\beta I}{N}+\mu+\gamma+\mu\right), J_{22}^{[2]}=-\left(\frac{\beta I}{N}+\mu+\bar{\alpha}+\frac{a b}{(b+I)^{2}}\right), J_{33}^{[2]}=$ $-\left(\frac{\beta I}{N}+\mu+\bar{k}\right), J_{44}^{[2]}=-\left(\gamma+\mu+\bar{\alpha}+a b /(b+I)^{2}\right), J_{55}^{[2]}=-(\gamma+\mu+\bar{k})$ and $J_{66}^{[2]}=-\left(\bar{\alpha}+\bar{k}+a b /(b+I)^{2}\right)$.

We choose $P(S, E, I, Q)=\operatorname{diag}[1 / E, 1 / I, 1 / Q, S / I, S / Q, S / Q]$, and we get that

$$
B=P_{f} P^{-1}+P J^{[2]} P^{-1}=\left[\begin{array}{cccccc}
M_{1} & \frac{\beta S I}{E N} & 0 & \frac{\beta I}{E N} & 0 & 0 \\
\frac{\gamma E}{I} & M_{2} & \frac{k Q}{I} & 0 & 0 & 0 \\
0 & \alpha_{q} I / Q & M_{3} & 0 & 0 & -\beta / N \\
0 & \frac{\beta S I}{N} & 0 & M_{4} & \frac{k Q}{I} & 0 \\
0 & 0 & \frac{\beta S I}{N} & \frac{\alpha_{q} I}{Q} & M_{5} & \frac{\beta S}{N} \\
0 & 0 & 0 & 0 & \gamma & M_{6}
\end{array}\right],
$$

where

$$
\begin{aligned}
& M_{1}=J_{11}^{[2]}-\frac{E^{\prime}}{E}, M_{2}=J_{22}^{[2]}-\frac{I^{\prime}}{I}, M_{3}=J_{33}^{[2]}-\frac{Q^{\prime}}{Q}, M_{4}=J_{44}^{[2]}+\frac{S^{\prime}}{S}-\frac{I^{\prime}}{I}, \\
& M_{5}=J_{55}^{[2]}+\frac{S^{\prime}}{S}-\frac{Q^{\prime}}{Q}, M_{6}=J_{66}^{[2]}+\frac{S^{\prime}}{S}-\frac{Q^{\prime}}{Q} .
\end{aligned}
$$

Now, we write $B$ as $B=\left[\begin{array}{ll}B_{11} & B_{12} \\ B_{21} & B_{22}\end{array}\right]$, where

$$
\begin{aligned}
& B_{11}=\left[\begin{array}{ccc}
M_{1} & \frac{\beta S I}{E N} & 0 \\
\frac{\gamma E}{I} & M_{2} & \frac{k Q}{I} \\
0 & \frac{\alpha_{q} I}{Q} & M_{3}
\end{array}\right], B_{12}=\left[\begin{array}{ccc}
\frac{\beta I}{E N} & 0 & 0 \\
0 & 0 & 0 \\
0 & 0 & \frac{-\beta}{N}
\end{array}\right], \\
& B_{21}=\left[\begin{array}{ccc}
0 & \frac{\beta S I}{N} & 0 \\
0 & 0 & \frac{\beta S I}{N} \\
0 & 0 & 0
\end{array}\right], B_{22}=\left[\begin{array}{ccc}
M_{4} & \frac{k Q}{I} & 0 \\
\frac{\alpha_{q} I}{Q} & M_{5} & \frac{\beta S}{N} \\
0 & \gamma & M_{6}
\end{array}\right] .
\end{aligned}
$$

Let $\mu(B)$ be the Lozinskii measure with respect to $|\cdot|_{\infty}$ norm defined by

$$
\mu(B)=\limsup _{h \rightarrow 0^{+}} \frac{|I+h B|_{\infty}-1}{h} .
$$


We have $\mu(B) \leq \sup \left\{g_{1}, g_{2}\right\}$, see [28], where $g_{1}=\mu_{\infty}\left(B_{11}\right)+\left|B_{12}\right|_{\infty}$ and $g_{2}=\left|B_{21}\right|_{\infty}+\mu_{\infty}\left(B_{22}\right)$ are matrix norms with respect to $l_{\infty}$ vector norm, and $\mu_{\infty}$ denotes the Lozinskii measure with respect to this $l_{\infty}$ norm. Thus, $\left|B_{12}\right|_{\infty}=\frac{\beta I}{E N}$ and $\left|B_{21}\right|_{\infty}=\frac{\beta S I}{N}$. And, to get $\mu_{\infty}\left(B_{11}\right)$ and $\mu_{\infty}\left(B_{22}\right)$, we add the absolute value of the non-diagonal elements to the corresponding diagonal element in each entire row, then the maximum of the diagonal elements will be the measure of the matrix, see [28]. Thus, we get $B_{11}^{\prime}$ and $B_{22}^{\prime}$ corresponding to $B_{11}$ and $B_{22}$, respectively, as follows:

$$
\begin{gathered}
B_{11}^{\prime}=\left[\begin{array}{ccc}
-\left(\frac{\beta I}{N}+\mu\right) & \frac{\beta S I}{E N} & 0 \\
\frac{\gamma E}{I} & \frac{a}{b+I}-\left(\frac{\beta I}{N}+\mu+\frac{a b}{(b+I)^{2}}\right) & \frac{k Q}{I} \\
0 & \frac{\alpha_{q} I}{Q} & -\left(\frac{\beta I}{N}+\mu\right)
\end{array}\right], \\
B_{22}^{\prime}=\left[\begin{array}{ccc}
X & \frac{k Q}{I} & 0 \\
\frac{\alpha_{q} I}{Q} & \frac{S^{\prime}}{S}+\frac{\beta S}{N}-\bar{\gamma} & \frac{\beta S}{N} \\
0 & \gamma & Y
\end{array}\right], X=\frac{S^{\prime}}{S}+\frac{a}{b+I}-\left(\bar{\gamma}+\frac{a b}{(b+I)^{2}}+\frac{\gamma E}{I}\right), \\
Y=\frac{S^{\prime}}{S}+\gamma-\left(\bar{\alpha}+\frac{\alpha_{q} I}{Q}+\frac{a b}{(b+I)^{2}}\right) . \text { Thus, } \\
\mu_{\infty}\left(B_{11}\right)=\frac{a}{b+I}-\left(\frac{\beta I}{N}+\mu+\frac{a b}{(b+I)^{2}}\right), \quad \mu_{\infty}\left(B_{22}\right)=\frac{S^{\prime}}{S}+\frac{\beta S}{N}-\bar{\gamma} .
\end{gathered}
$$

It follows that

$$
\begin{aligned}
g_{1} & =\mu_{\infty}\left(B_{11}\right)+\left|B_{12}\right|_{\infty}=\frac{a}{b+I}-\left(\frac{\beta I}{N}+\mu+\frac{a b}{(b+I)^{2}}\right)+\frac{\beta I}{E N} \\
& =\frac{S^{\prime}}{S}+\frac{a}{b+I}+\frac{\beta I}{E N}-\frac{\mu N}{S}-\frac{a b}{(b+I)^{2}} \text { and } \\
g_{2} & =\left|B_{21}\right|+\mu_{\infty}\left(B_{22}\right)=\frac{\beta S I}{N}+\frac{S^{\prime}}{S}+\frac{\beta S}{N}-\bar{\gamma}=\frac{S^{\prime}}{S}+\frac{\beta S I}{N}+\frac{\beta S}{N}-\bar{\gamma} .
\end{aligned}
$$

It can be verified that $\sup \left\{g_{1}, g_{2}\right\}=g_{2}<0$ for $\mathcal{R}_{t}>1$. Hence,

$$
\bar{q}_{2} \leq \limsup _{t \rightarrow \infty} \sup _{x_{0} \in K} \frac{1}{t} \int_{0}^{t} g_{2} d t<0,
$$

implying that condition (iv) is satisfied. Therefore, $X_{2}^{*}$ is globally asymptoticaly stable provided that $\mathcal{R}_{t}>1$. 